\title{
Die Proteom-Signatur verändert sich mit dem Fortschreiten der chronischen myeloischen Leukämie (CML)
}

Kennzeichnend für die CML ist eine konstitutive Erhöhung der BCR-ABL1Kinase-Aktivität, die durch verschiedene Tyrosinkinaseinhibitoren (TKI) gehemmt werden kann. Bei Progression der Erkrankung kommt es jedoch häufig zu einer zunehmenden Resistenz gegenüber den ansonsten effektiven TKI. Die biologischen Grundlagen sind noch nicht ausreichend verstanden.

$\mathrm{M}$ ithilfe von Reverse-Phase-ProteinArrays (RPPA) und 112 individuellen monoklonalen Antikörpern wurden die Proteinexpressionsmuster von 40 Patienten mit CML (25 in der chronischen, 5 in der akzelerierten Phase und 10 in der Blastenkrise) untersucht. Die Varianzanalyse zwischen den drei Phasen der CML ergab ein Set von Proteinen, die in der Blastenkrise signifikant überexprimiert waren: HeatshockProtein 90 (hsp90); Retinoblastom-Protein (Rb); Apoptose-induzierender Faktor (AIF); Serin/Threonin-Protein-Phosphatase 2A (PP2A); B-Zell-LeukämieProtein 2 (Bcl-2); X-linked inhibitor of
apoptosis-Protein (Xiap); weiter die Proteine Mad, Smad1, SSBP2a, PARP, Gab2 und Trim24. Mehrere dieser Proteine waren auch im Kompartiment der CD34-positiven CML-Stammzellen überexprimiert.

Ein Vergleich mit der jeweils zugehörigen Expression der mRNA-Transkripte zeigte, dass von zwölf in den RPPA überexprimierten Proteinen in der Blastenphase zehn auch auf mRNA-Ebene erhöht waren. Ähnliche (inkomplette) Überlappungen zwischen Protein- und mRNA-Überexpression zeigten sich für die CD34-positiven Zellen. Umgekehrt konnten auch Proteine identifiziert wer- den, die in der Blastenphase der CML bzw. in den nicht-CD34-positiven Zellen geringer exprimiert waren als in den jeweiligen Vergleichskompartimenten.

Fazit: Die Autoren um Alfonso QuintásCardama folgern, dass einige der in den fortgeschrittenen Phasen der CML zunehmend exprimierten Proteine geeignete Targets für die Entwicklung zielgerichteter Medikamente sein könnten. Das gilt auch für die ruhenden CMLStammzellen, die von TKI nicht erfasst werden und wohl dafür verantwortlich sind, dass nur eine begrenzte Zahl von Patienten eine komplette molekulare Remission erzielt.

Brigitte Schalhorn

Quintás-Cardama A et al. Reverse phase protein array profiling reveals distinct proteomic signatures associated with chronic myeloid leukemia progression and with chronic phase in the CD34-positive compartment. Cancer. 2012; 118(21):5283-92.

\section{Im Vergleich: TKI der ersten und zweiten Generation gegen CML}

Die Einführung des Tyrosinkinaseinhibitors (TKI) Imatinib (IM) markierte einen Meilenstein in der Behandlung der chronischen myeloischen Leukämie (CML). Patienten mit Unverträglichkeit oder Resistenz gegen IM profitieren von einem TKI der zweiten Generation.

$\mathrm{n}$ der IRIS (International Randomized Study of Interferon and STI571)-Studie führte die Behandlung mit $400 \mathrm{mg}$ IM

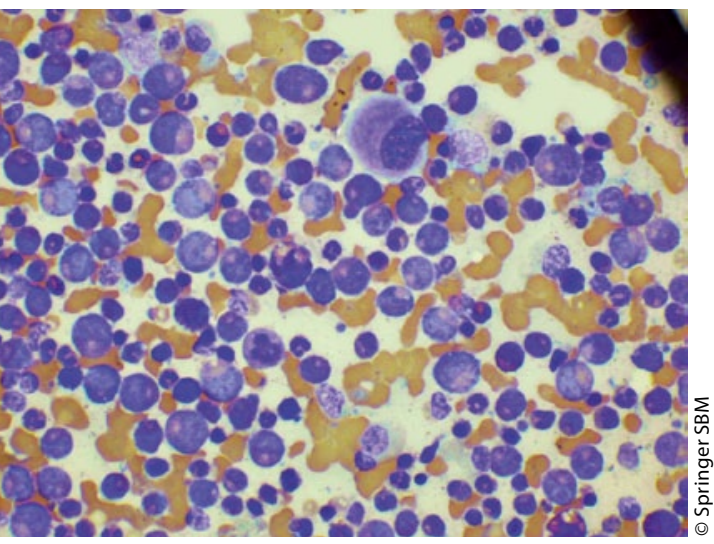

Knochenmarkbefund eines CML-

Patienten mit gesteigerter Granulopoese. pro Tag nach zwölf Monaten bei $69 \%$ der Patienten zur vollständigen zytogenetischen Remission (CCyR). Jedoch schieden $31 \%$ der Patienten vor allem wegen inakzeptabler Toxizität oder Nichtansprechen vorzeitig aus dem IM-Arm aus.

Mit dem Multikinaseinhibitor Dasatinib (DAS) können bei diesen Patienten CCyR-Raten von rund $40 \%$ erzielt werden. Auch die molekularen und zytogenetischen Remissionsraten sind gegenüber IM höher und die Progressionstendenz ist geringer. Die vorliegende Arbeit präsentiert die Ergebnisse der 2005 gestarteten S0325-Studie, in der $400 \mathrm{mg} / \mathrm{d}$ IM und $100 \mathrm{mg} / \mathrm{d}$ DAS randomisiert bei 253 CML-Patienten in chronischer Phase verglichen wurden.

Eine CCyR wurde in der DAS-Gruppe von deutlich mehr Patienten erreicht als im Vergleichsarm (84 vs. 69\%). Auch molekulare Remissionen mit einer 3-log, 4-log- oder 4,5-log-Reduktion der BCRABL-Transkripte waren häufiger unter DAS. Keine signifikanten Unterschiede ergaben sich im gesamten und progressionsfreien Überleben (3-Jahres-PFS von $91 \%$ unter DAS und $88 \%$ unter IM). Toxizitäten vom Grad 3/4 waren meist hämatologisch und unter DAS häufiger (18 vs. $8 \%)$.

Fazit: Patienten mit CML in chronischer Phase profitieren vom ZweitgenerationsTKI Dasatinib in Form eines früheren zytogenetischen und molekularen Ansprechens im Vergleich zu Imatinib. Der Vorteil wird mit erhöhter hämatologischer Toxizität erkauft, beide Wirkstoffe sind ähnlich effizient bezüglich des Gesamtüberlebens.

Wolfgang Zimmermann

Radich JP et al. A randomized trial of dasatinib $100 \mathrm{mg}$ versus imatinib $400 \mathrm{mg}$ in newly diagnosed chronic myeloid leukemia. Blood. 2012; 120(19):3898-905. 